\title{
Mechanical characterization of nanostructured thin films at different scales
}

\author{
S. Djaziri ${ }^{1}$, D. Thiaudière ${ }^{2}$, G. Geandier ${ }^{2}$, P.-O. Renault ${ }^{1}$, E. Le Bourhis ${ }^{1}$, P. Goudeau ${ }^{1}$, R.N.

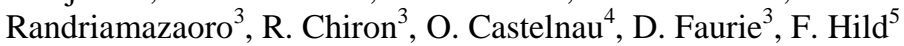 \\ ${ }^{1}$ Institut P' CNRS - Université de Poitiers - ENSMA, UPR 3346, 86962 Futuroscope, France \\ ${ }^{2}$ Synchrotron SOLEIL, L'Orme des Merisiers, BP 48, 91192 Gif sur Yvette, France \\ ${ }^{3}$ LPMTM, UPR 9001 CNRS, Université Paris-Nord, 93430 Villetaneuse, France \\ ${ }^{4}$ Laboratoire PIMM, ENSAM, 151, Boulevard de l'Hôpital, 75013 Paris, France \\ ${ }^{5}$ LMT Cachan, 61 Avenue du Président Wilson 94235 CACHAN Cedex, France
}

\begin{abstract}
The mechanical behaviour of multilayered $\mathrm{W} / \mathrm{Cu}$ thin films on polyimide substrate has been investigated at different scales and using complementary X-ray techniques and compared to finite element analysis. Mechanical testing has been carried out using a new biaxial tensile machine which allows for testing in controlled biaxial loading condition. This device has been developed for synchrotron measurements at DiffAbs beamline of the French synchrotron radiation facility (SOLEIL, Saint Aubin).
\end{abstract}

\section{Introduction}

Due to interesting characteristics, such as flexibility and low thickness, thin metal films on polymer substrates are becoming increasingly used in many technological applications such as stretchable microelectronics [1-2], polymer metallization [3] and aeronautics. Enhanced properties can be obtained by combining different materials in form of nanostructured thin film [4]. In particular, tungsten-copper $(\mathrm{W}-\mathrm{Cu})$ composites show very important mechanical properties resulting from the compromise between the high strength of $\mathrm{W}$ and the ductility of $\mathrm{Cu}$ [5-7]. Owing to the specific fabrication, nanoscaled materials present physical properties which are different to bulk counterparts [8] and are subjected to high stresses that can lead to the formation of damages such as cracks and surface delamination in the case of metallic films. Thus, understanding the mechanical behaviour of nanostructured thin films is of utmost importance for ensuring the reliability of systems. Amongst specialized techniques for mechanical characterization of supported thin films, in-situ tensile testing has been proven to be the most suitable method [9-16]. Uniaxial testing commonly used generates a non-equi-biaxial state in the film due to Poisson's ratios mismatch between the substrate and the metallic thin film. As described and validated in a very recent work [17], the new biaxial tensile machine, which has been developed for DiffAbs beamline at SOLEIL, the French synchrotron facility (Saint Aubin), allows for testing in both equi-biaxial and non-equi-biaxial loading conditions, mimicking in a more realistic manner the complex stress state applied to nanostructured systems during service in many application fields. Noticeably, bulge and ring on ring tests can be used to investigate the behaviour of thin films under plane stresses [18-19]. Due to the thermal expansion mismatch between film and substrate, a biaxial stress state can also be applied to the film

This is an Open Access article distributed under the terms of the Creative Commons Attribution-Noncommercial License 3.0, which permits unrestricted use, distribution, and reproduction in any noncommercial medium, provided the original work is properly cited. 
by annealing the thin film/substrate samples at elevated temperatures [20-22]. However, these techniques are restricted to equi-biaxial loadings.

In this work, the tensile response of the $\mathrm{W}-\mathrm{Cu}$ composite deposited onto a polyimide cruciform substrate has been investigated under equi-biaxial loadings at different scales using complementary X-ray techniques: X-ray diffraction (XRD), X-ray reflectivity (XRR) and grazing incidence small angles X-ray scattering (GISAXS). The measured X-ray strains in tungsten grains have been compared to the global strains calculated by finite element method.

\section{Design of the biaxial tensile machine}

The tensile machine has been designed to allow loading along two normal axes cruciform substrates coated by the studied films. The machine is composed of four identical module components. Each module contains a motor, a force sensor and a cylindrical fixation. The weight of the device is $3.5 \mathrm{~kg}$ and its size is $19 \times 19 \times 8.5 \mathrm{~cm}^{3}$ with a free space at the centre. The cruciform substrates were coated at their centre only and gripped by a cam rotating in the cylinder fixation. The machine can apply forces up to $200 \mathrm{~N}$. A polymeric substrate is chosen to minimize its mechanical contribution to the total response of the film / substrate set since we are interested here in the film mechanical behaviour. Furthermore, the polyimide substrate is expected to behave elastically in the investigated strain range for metallic film (maximum of $0.4 \%$ ). Here, we used $125-\mu \mathrm{m}$-thick polyimide substrate (sofimide® from MICEL). Fig. 1 shows the machine with a gripped cruciform specimen.

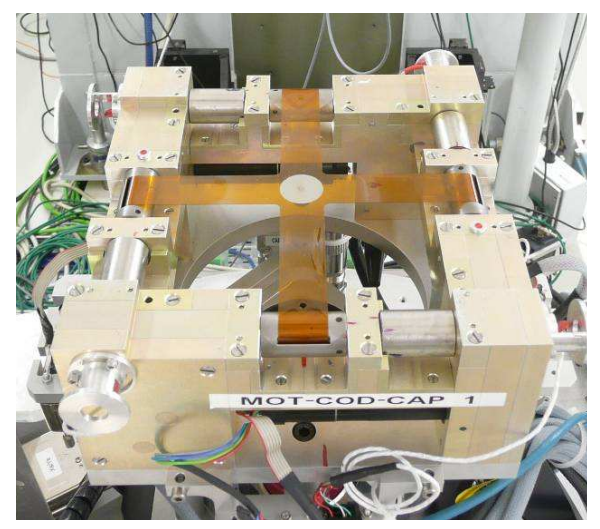

Fig. 1. The biaxial tensile machine with a gripped cruciform specimen. The W/Cu thin film can be seen at the centre of the sofimide substrate.

\section{Strains measurements}

Mechanical behaviour of the specimen was obtained at two different scales. At the macro-scale, finite element (FE) analysis was used to design and model the cruciform substrate mechanical behaviour for biaxial loadings. Owing to different X-ray techniques, in-situ biaxial tensile testing of the coated cruciform specimen allowed measuring strains at the nano-scale.

\subsection{Global strain}

Using a new experimental set-up requires the design of the cruciform specimen geometry. In order to achieve homogeneous stress condition on a few square millimetres, the specimen geometry is optimised based on FE-simulation. Indeed, the required substrate geometry must allow obtaining small deformations (to study the elastic domain) within an area of the specimen centre wide enough to achieve X-ray measurements. The mechanical behaviour of the polyimide cruciform substrate has 
been modelled using the CAST3M software from the French atomic energy commission (CEA in French). The branches of the chosen specimen are $20 \mathrm{~mm}$ in width with $5 \mathrm{~mm}$ toe weld as illustrated in Fig. 2.

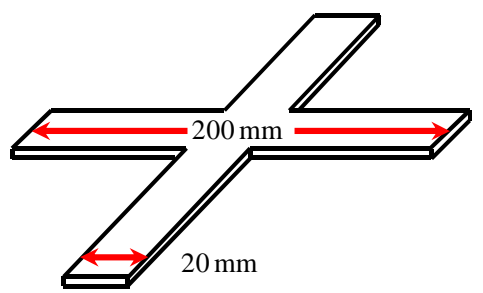

Fig. 2. Schematic representation of the cruciform substrate.

Two-dimensional FE-calculations were performed under plane-stress conditions because of the specimen's small thickness. Besides, only the quarter of the structure was modelled for axial symmetry reasons. A linear elastic behaviour was adopted to assess the mechanical behaviour of the cruciform substrate loaded biaxially. This represents a good approximation of the true behaviour of the specimen since small strains involve, in general, linear stress-strain relation as experimentally determined in uniaxial tensile tests. Actually, strain measurements by digital image correlation for sofimide ${ }^{\circledR}$ revealed a polynomial form of the stress-strain curves as reported by D. Y. W. Yu and F. Spaepen for a similar polyimide (supplied by Dupont de Nemours and named Kapton®) [23]. The behaviour is linear up to a strain of $0.8 \%$, a value below which the experimental study was carried out. Young's modulus $\left(\mathrm{E}_{\mathrm{s}}\right)$ and Poisson's ratio $\left(v_{\mathrm{s}}\right)$ were determined to be $\mathrm{E}_{\mathrm{s}}=2.7 \pm 0.1 \mathrm{GPa}$ and $v_{\mathrm{s}}=0.34$ respectively in this deformation range, values that were used in FE-calculations.

The strain distributions of the two components $\varepsilon_{\mathrm{xx}}$ and $\varepsilon_{\mathrm{yy}}$ in the specimen under a $40 \mathrm{~N}$ equi-biaxial loading are illustrated in Fig. 3a and Fig. $3 \mathrm{~b}$ respectively. Due to the symmetry of the specimen, those two figures are the same by a rotation of $90^{\circ}$. The centre of the specimen seems to exhibit a region in which the strain is homogeneous. This is checked thanks to sections along three different axes (Fig. 3).

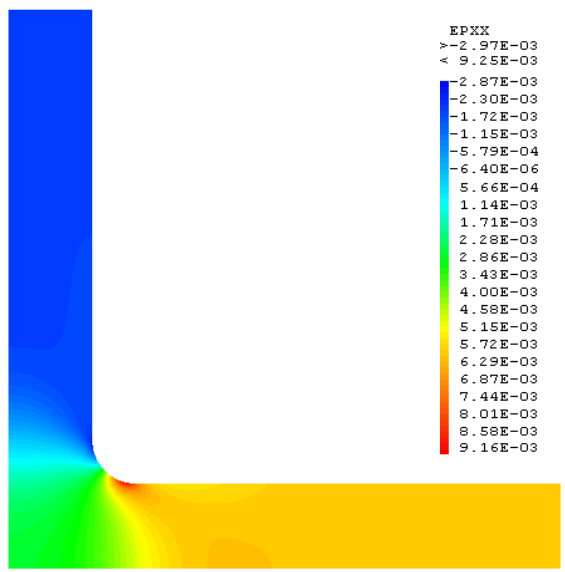

a)

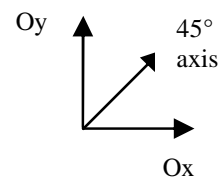

Fig. 3. FE strain field of a cruciform substrate under a $40 \mathrm{~N}$ equi-biaxial loading a) $\varepsilon_{\mathrm{xx}}$ and b) $\varepsilon_{\mathrm{yy}}$.

Strain components along the axis at $45^{\circ}$ are equal and remain constant over a distance longer than 6 $\mathrm{mm}$ (Fig. 4a). Along Ox and Oy axes, the distance reduces to about $4 \mathrm{~mm}$. As shown in Fig. 4b, the strain components along $\mathrm{Ox}$ axis (at the right of the figure) are equal for roughly $2 \mathrm{~mm}$ in distance. Similarly, they are equal to a distance of about $2 \mathrm{~mm}$ along Oy axis (at the left of the figure). Hence, 
the strain field can be considered as uniform in the centre of the cruciform specimen over a distance of $4 \mathrm{~mm}$. We conclude, according to the FE analysis, that for a $40 \mathrm{~N}$ equi-biaxial loading, a $0.27 \%$ homogeneous strain is generated in a central area of $4 \mathrm{~mm}$ in radius for the chosen geometry.

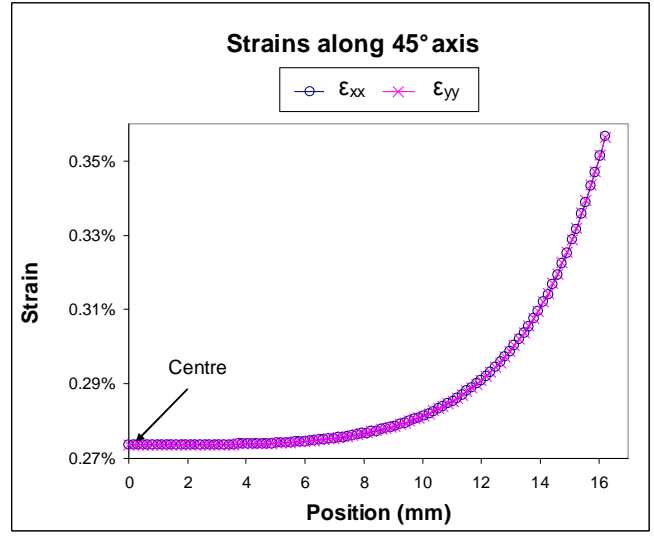

a)

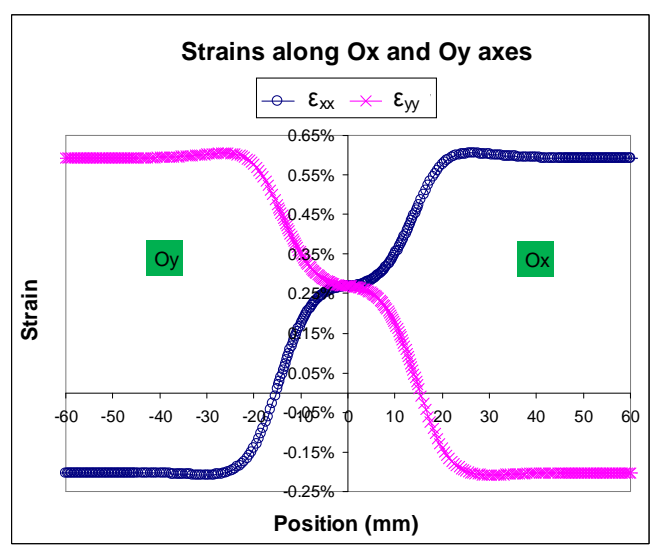

b)

Fig. 4. Strain components variations under a $40 \mathrm{~N}$ equi-biaxial loading along a) $45^{\circ}$ axis and b) $\mathrm{Ox}$, $\mathrm{Oy}$.

\subsection{X-ray strain}

We have investigated the mechanical behaviour of $\mathrm{W} / \mathrm{Cu}$ thin films deposited onto polyimide substrate (sofimide ${ }^{\circledR}$ from MICEL). The thin films were produced at room temperature by physical vapour deposition (PVD) with an Ar+-ion-gun sputtering beam at $1.2 \mathrm{keV}$. The base pressure of the deposition chamber was $7 \times 10^{-5} \mathrm{~Pa}$ while the working pressure during film growth was approximately $10^{-2} \mathrm{~Pa}$. The used cruciform substrate is $125 \mu \mathrm{m}$ in thickness. The thin film comprises 37 periods of $4 \mathrm{~nm}$ each, composed of $3 \mathrm{~nm} \mathrm{~W}$ and $1 \mathrm{~nm} \mathrm{Cu}$. The total film thickness was evaluated to be about $150 \mathrm{~nm}$. Crystallographic texture analysis was carried out using XRD and it was shown that $\mathrm{W}$ constituent of $\mathrm{W} / \mathrm{Cu}$ composites exhibits a strong $\{110\}$-fibre texture. Global residual stress of the film determined by Stoney curvature method was found to be $-1.5 \mathrm{GPa}$ while $\mathrm{W}$ grain residual stress analysed by XRD using the $\sin ^{2} \psi$ method was equal to -4 GPa [24].

In-situ tensile tests have been carried out at SOLEIL synchrotron. Indeed, X-ray synchrotron radiation allows faster and more accurate measurements as it allows studying small diffracting volumes of material with high resolution [25]. Strain measurements have been performed for an equi-biaxial loading using different X-ray techniques. An initial load has to be applied for the installation of the specimen into the biaxial tensile device in order to avoid sample drift during the biaxial tensile test. In the following, the applied loads are increments from the initial loading state, and are ranged from $0 \mathrm{~N}$ to $40 \mathrm{~N}$. The X-ray energy and beam size have been set to around $8 \mathrm{keV}$ and $0.300 \times 0.250 \mathrm{~mm}^{2}(\mathrm{FWHM}, \mathrm{H} \times \mathrm{V}$ respectively).

\subsubsection{In-grain strain}

$\mathrm{X}$-ray diffraction has been used to measure the intra-granular strain of W constituent. It was obtained by detecting the diffraction peak position shift for each applied load. During the test, the incremental increase of applied load was halted to allow for the X-ray diffraction measurements. The measurements have been performed for different $\psi$ angles and along two normal directions $\phi=0$ and $\phi=90^{\circ}$. As shown in Fig. $4, \psi$ is the angle between the specimen surface normal $\mathrm{S}_{3}$ and the diffracting plane normal. $\phi$ is the rotation angle of the specimen around its surface normal. $S_{1}$ and $S_{2}$ are the loading directions. 


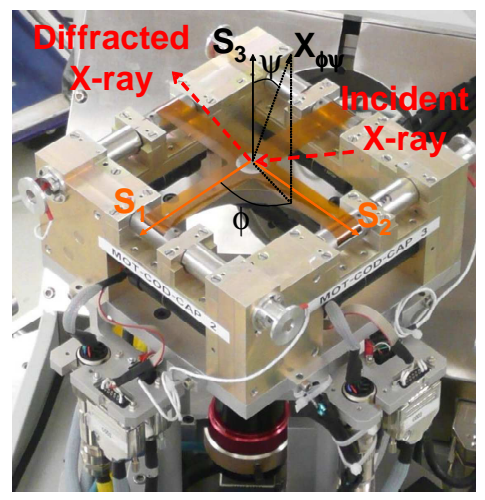

Fig. 5. Experimental configuration of the tests.

Diffraction data were acquired for $\mathrm{W}-\{211\}$ crystallographic planes due to accuracy improvement on strain measurements at higher Bragg angles. For an equi-biaxial loading, the strain $\varepsilon_{\psi}$ along the two axes is given by [26]:

$$
\varepsilon_{\psi}=\left(\frac{1+v}{\mathrm{E}}\right) \bar{\sigma} \sin ^{2} \psi-\frac{2 v}{\mathrm{E}} \bar{\sigma}
$$

Where $\bar{\sigma}$ is the macroscopic stress applied to the thin film. E is the Young's modulus and $v$ the Poisson's ratio of the material. This expression reflects the $\sin ^{2} \psi$ law which describes a linear relationship between $\varepsilon$ and $\sin ^{2} \psi$ characteristic of locally isotropic materials such as W [27]. Elastic strain measured by XRD is plotted as a function of $\sin ^{2} \psi$. All measured curves shown in Fig. 6 are linear as expected for a locally isotropic material. For the equi-biaxial loading, we observed that elastic strains are similar along both directions $\left(\phi=0^{\circ}\right.$ and $\left.\phi=90^{\circ}\right)$. Furthermore, all straight lines intersect at a point in the vicinity of zero strain which allows determining Poisson's ratio of the film. The obtained value is close to the bulk one (0.285). Extracting the slope and intercept of $\varepsilon-\sin ^{2} \psi$ plots, we can determine the components of the strain tensor. Actually, in the case of an equi-biaxial loading, both principal strain components are identical and equal to the sum of the slope and intercept of $\varepsilon-\sin ^{2} \psi$ curves.

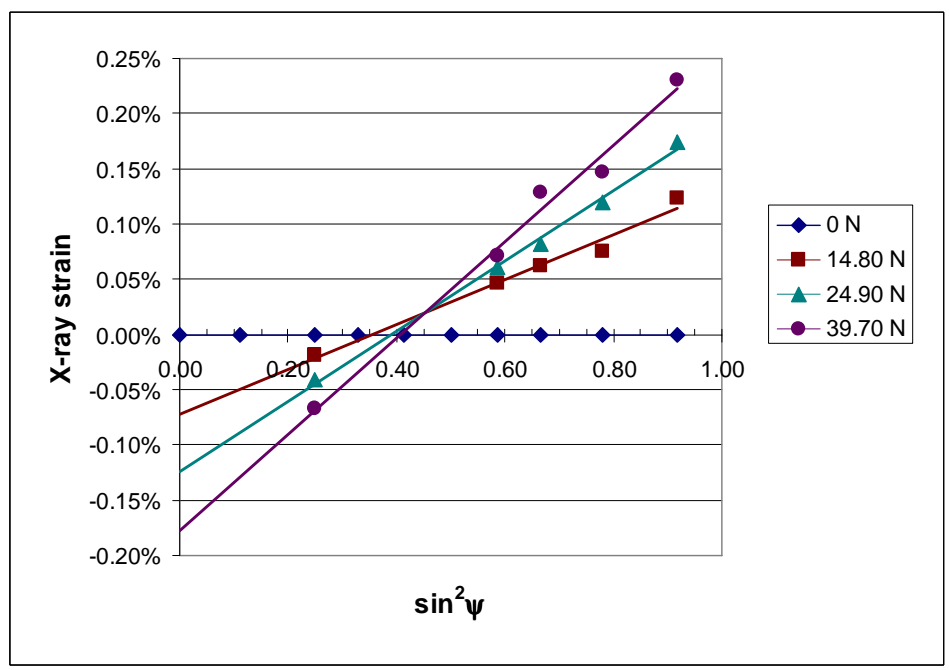

Fig. 6. W X-ray strain as a function of $\sin ^{2} \psi$ for $\phi=0^{\circ}$. 
The obtained value for a $40 \mathrm{~N}$ equi-biaxial loading is $0.27 \pm 0.02 \%$ as predicted by finite elements analysis. This shows that a complete strain transfer can be assumed through the interface between the polyimide substrate and the metallic thin film within the elastic domain. In comparison to the pure $\mathrm{W}$ thin film, it seems that the incorporation of $\mathrm{Cu}$ into a $\mathrm{W}$ matrix does not affect the $\mathrm{W}$ phase behaviour as long as the studied domain is in the elastic regime.

\subsubsection{Out-of-plane strain}

Thanks to the biaxial tensile machine, X-ray reflectometry (XRR) can be achieved on the cruciform specimen. These measurements are carried out at very low angles. Indeed, the cylinder fixations of sample branches do not disturb measurements at grazing incidence. Moreover, the surface of specimens becomes flat enough upon loading, allowing XRR measurements. This technique was used to determine at the multilayer period scale the out-of-plane strain since XRR quantifies film thicknesses, multilayer periodicity, surface and interface roughness. Fig. 7 shows the specular X-ray reflectance of the periodic $\mathrm{W} / \mathrm{Cu}$ multilayer; the presence of three diffraction peaks clearly shows the existence of a multilayer periodicity.

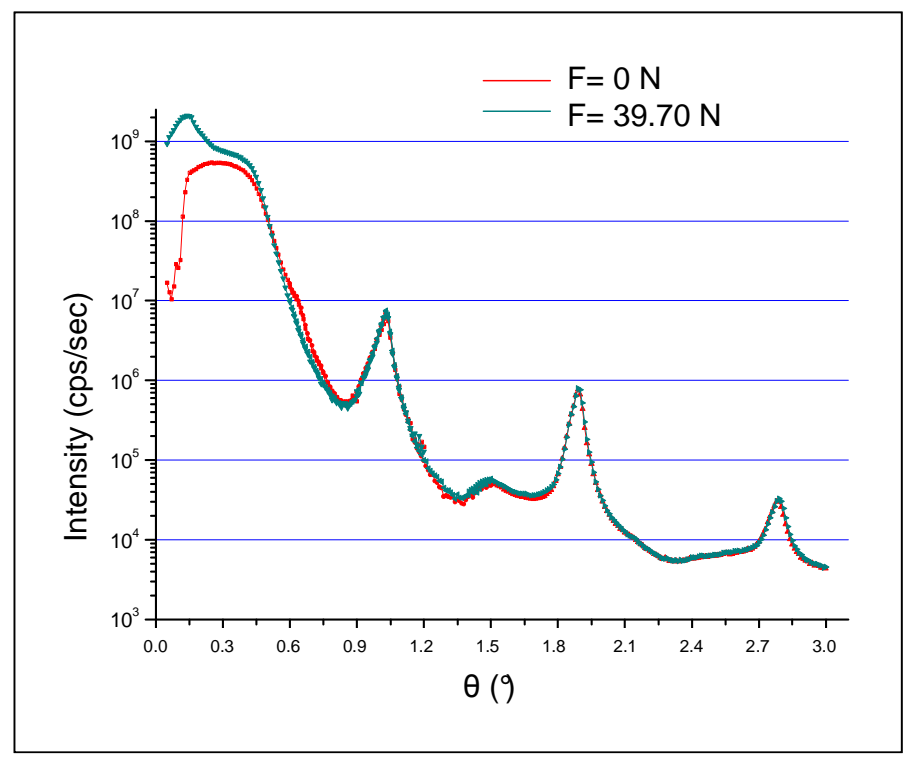

Fig. 7. X-ray reflectometry curves measured for W/Cu multilayers during in situ deformation.

By matching the positions and relative intensities of the Bragg peaks at low angles, the bilayer thickness was determined to be $4.49 \mathrm{~nm}$ which is slightly higher than the nominal thickness of the specimen. The vertical deformation of bilayers for a loading increment of $39.70 \mathrm{~N}$ is relatively small (1/3 of the in plane strain due to Poisson's ratio). However, we show that the out-of-plane deformation of $\mathrm{W} / \mathrm{Cu}$ bilayers at the period scale can be measured with this technique. For stronger applied forces, the shift should be more visible.

\subsubsection{In-plane strain}

Grazing Incidence Small Angles X-ray Scattering (GISAXS) technique is very useful to characterize the in-plane structure and morphology of $\mathrm{W} / \mathrm{Cu}$ composites or equivalent systems. Especially, it 
allows obtaining the size of $\mathrm{Cu}$ nanoparticles embedded into the $\mathrm{W}$ matrix as well as the distribution of these clusters.

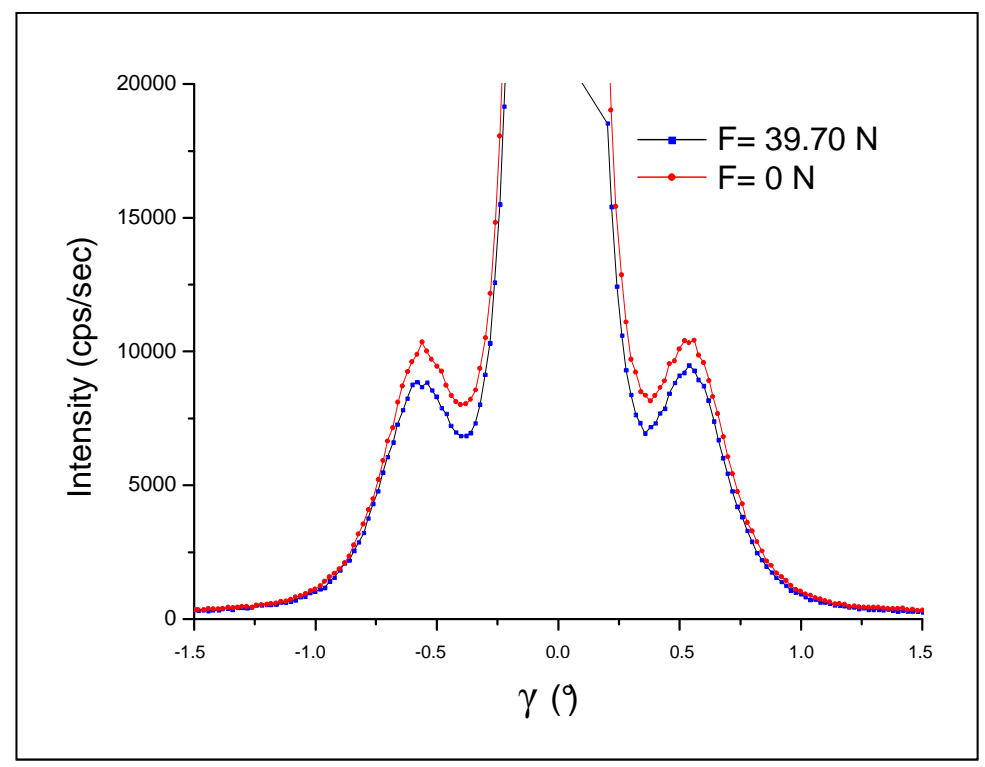

Fig. 8. GISAXS for W/Cu multilayers.

As illustrated in Fig. 8, the copper layer is discontinuous and the film can be assimilated to cluster dispersion in a tungsten matrix. The correlation distance between nanoparticles given by the position at maximum intensity has increased between the loading increments $F=0 \mathrm{~N}$ and $\mathrm{F}=39.70 \mathrm{~N}$ from $15.2 \mathrm{~nm}$ up to $15.7 \mathrm{~nm}$. The measured in plane strain is then about $0.3 \%$, a value close to the XRD maximum strain. This shows that the $\mathrm{Cu}$ component of the multilayer is deformed under the applied loading in the same way as in grain W diffracting planes. Let us notice that the in-plane strain corresponds here to the strain measured at the $\mathrm{Cu}$ nanoparticles scale.

\section{Conclusions}

In this study, we have investigated the mechanical behaviour of the $\mathrm{W}$ component of the $\mathrm{W} / \mathrm{Cu}$ multilayered thin film deposited on polyimide substrate for a controlled biaxial loading. X-ray techniques allowed characterizing the thin film at different scales thanks to the synchrotron radiation. The dedicated biaxial tensile device can be used to induce a biaxial uniform strain field in a section of about $4 \times 4 \mathrm{~mm}^{2}$ in the centre of the coated substrate both under equi-biaxial and nonequi-biaxial loadings. The measured X-ray strain is in a good agreement with the strain calculated by finite element analysis. XRD alone is not sufficient to get all the structural information required to determine mechanical behaviour of $\mathrm{W} / \mathrm{Cu}$ nanocomposite systems. The use of different X-ray techniques such as reflectometry and grazing incidence small-angle X-ray scattering (GISAXS) is promising for strain measurements in case of nanostructured composites. For complete structural information, extended X-ray absorption fine structure (EXAFS) spectroscopy should be performed near $\mathrm{Cu} \mathrm{K}$ absorption edge to follow strains in the copper clusters since this technique is very sensitive to local order while diffracting methods are not appropriated. 


\section{References}

1. M.A. Meyers, A. Mishra, D.J. Benson, Prog. Mater. Sci. 51, 427 (2006).

2. S.P. Lacour, D. Chan, S. Wagner, T. Li, Z. Suo, Appl. Phys. Lett. 88, 204103 (2006).

3. W.T. Li, R.B. Charters, B. Luther-Davies, L. Mar, Appl. Surf. Sci. 233, 227 (2004).

4. P.A. Gruber, E. Arzt, R. Spolenak, J. Mater. Res. 24, 1906 (2009).

5. A. Luedtke, Adv. Eng. Mater. 6, 142 (2004).

6. M. Gassik, Comput. Mater. Sci. 13, 42 (1998).

7. P. M. Geffroy, T. Chartier, J. F. Silvain, Ann. Rev. Mater. Sci. 9, 547 (2007).

8. F. Spaepen, D.W.Y. Yu, Scr. Mater. 50, 729 (2004).

9. P.-O. Renault, K.F. Badawi, L. Bimbault, Ph. Goudeau, E. Elkaïm, J.P. Lauriat, Appl. Phys. Lett. 73, 1952 (1998).

10. O. Kraft, M. Hommel, E. Arzt, Mater. Sci. Eng., A 288, 209 (2000).

11. K.F. Badawi, P. Villain, Ph. Goudeau, and P.-O. Renault, Appl. Phys. Lett. 80, 4705 (2002).

12. D. Faurie, P.O. Renault, E. Le Bourhis, P. Villain, Ph. Goudeau, F. Badawi, Thin Solid Films, 201, 469 (2004).

13. D. Faurie, P.O. Renault, E. Le Bourhis, Ph. Goudeau, O. Castelnau, R. Brenner, G. Patriarche, Appl. Phys. Lett. 89, 061911 (2006).

14. I.C. Noyan, G. Sheikh, J. Mater. Res. 8, 8 (1992).

15. Kretschmann, W.-M. Kuschke, S.P. Baker and E. Arzt, Mater. Res. Soc. Symp. Proc. 436, 59 (1996).

16. I.C. Noyan, G. Sheikh, Mater. Res. Soc. Symp. Proc. 308, 3 (1993).

17. G. Geandier and al, (submitted to Rev. Sci. Instrum).

18. J.J. Vlassak and W.D. Nix, J. Mater. Res. 7, 3242 (1992).

19. S. Eve, N. Hubert, O. Kraft, A. Last, D. Rabus, M. Schlagenhof, Rev. Sci. Instr. 77, 103902 (2006).

20. G. Cornella, S.-H. Lee, W.D. Nix, J.C. Bravman, Appl. Phys. Lett. 71, 2949 (1997).

21. S.P. Baker, A. Kretschmann, E. Arzt, Acta. Mater. 49, 2145 (2001).

22. J. Keckes, J. Appl. Cryst. 38, 311 (2005).

23. D.W.Y. Yu, F. Spaepen, J. Appl. Phys. 95, 2991 (2004).

24. B. Girault, PhD thesis of Poitiers University (2008), France.

25. G. Geandier, P.-O. Renault, S. Teat, E. Le Bourhis, B. Lamongie and Ph. Goudeau, J. Appl. Cryst., 41, 1076 2008).

26. V. Hauk, Structural and Residual Stress Analysis by Non destructive Methods: Evaluation, Application, Assessment (Elsevier, Amsterdam, 1997).

27. D. Faurie, O. Castelnau, R. Brenner, P.-O. Renault, E. Le Bourhis, Ph. Goudeau, J. Appl. Cryst., 42, 1073 (2009). 